\title{
A Contemporary Risk Analysis of Iatrogenic Transmission of Creutzfeldt-Jakob Disease (CJD) via Corneal Transplantation in the United States
}

\author{
Tanisha Martheswaran · Jordan D. Desautels • Majid Moshirfar (D) · \\ Kathryn M. Shmunes · Yasmyne C. Ronquillo · Phillip C. Hoopes
}

Received: May 13, 2020 / Published online: June 20, 2020

(C) The Author(s) 2020

\section{ABSTRACT}

Background: Creutzfeldt-Jakob disease (CJD) is a rare, fatal, neurodegenerative prion disease potentially transmissible through corneal transplantation. While statistical analyses performed two decades ago estimated the overall prevalence of CJD in the corneal donor pool to be low, the recent significant increase in corneal

Digital Features To view digital features for this article go to https://doi.org/10.6084/m9.figshare.12459605.

Electronic supplementary material The online version of this article (https://doi.org/10.1007/s40123020-00272-8) contains supplementary material, which is available to authorized users.

\section{T. Martheswaran}

Department of Stem Cell and Regenerative Biology, Harvard University, Cambridge, MA, USA

J. D. Desautels

The Warren Alpert Medical School of Brown

University, Providence, RI, USA

M. Moshirfar $(\bowtie) \cdot$ K. M. Shmunes .

Y. C. Ronquillo · P. C. Hoopes

Hoopes Vision Research Center, Draper, UT, USA

e-mail: Cornea2020@me.com

\section{Moshirfar}

Department of Ophthalmology and Visual Sciences, John A. Moran Eye Center, University of Utah

School of Medicine, Salt Lake City, UT, USA

M. Moshirfar

Utah Lions Eye Bank, Murray, UT, USA transplants performed and deaths due to CJD in the U.S. warrants a contemporary risk analysis. Methods: A literature review was conducted to determine the overall number of globally reported cases of CJD transmission through corneal transplantation. U.S. mortality and cornea donation data were utilized to estimate the age-stratified prevalence of undiagnosed, latent CJD in the cornea donor pool in 2018. A historical statistical analysis was performed to estimate the number of corneas from donors with latent CJD entering the U.S. donor pool for each year between 1979 and 2018. From these statistical analyses, risk factors of iatrogenic transmission were identified and summarized.

Results: Ten reported cases of iatrogenic transmission of CJD through corneal transplants were identified globally. In 2018, an estimated 3.8 corneas from donors with undiagnosed latent CJD potentially entered the pool of 111,703 transplant-intended corneas harvested from individuals aged 31-80. Between 1979 and 2018, an estimated 47 corneas may have entered the U.S. transplant-intended pool from donors with latent CJD aged 35 to 84 . The advanced age of donors and a history of multiple transplants in recipients were both prominent risk factors for iatrogenic transmission.

Conclusions: The 10 reported global cases of iatrogenic transmission likely under-represent the number of individuals with a coinciding history of death by CJD and prior corneal transplantation, as supported by our statistical 
analysis and lack of geographical diversity of reported cases. As effective screening methods develop and globalization of cornea transplantation broadens, it is of utmost importance that cornea transplantation history among victims of CJD should be investigated and reported.

Keywords: CJD; Cornea; Creutzfeldt-Jakob disease; Eye; Iatrogenic; Ophthalmology; Risk analysis; Spongiform encephalopathy; Transmission; Transplant

\section{Key Summary Points}

\section{Why carry out this study?}

Iatrogenic transmission of CreutzfeldtJakob disease (CJD) has been shown to occur through corneal transplantation in animals, with 10 reported cases of suspected transmission among humans.

The last comprehensive statistical analysis of iatrogenic transmission of CJD through corneal transplantation was performed two decades ago. Since then, mortality due to CJD has more than doubled, and corneal transplantation has increased steadily. Our team deemed it relevant to conduct an updated analysis of this lifethreatening occurrence.

Conduct a contemporary and historical statistical analysis, estimating prevalence of CJD-infected corneas in both modernday and historical transplant pools.

Identify donor and recipient risk of transmission of CJD through corneal transplantation.

\section{What was learned from the study?}

From our analyses, it is estimated that 1 in every 30,000 transplants in the U.S. will be performed with a cornea from a donor with latent, undiagnosed CJD.
Old age of donors and history of multiple transplants in recipients, especially with tissue from elderly donors, are both prominent risk factors for transmission of CJD through corneal transplantation.

Potential cases are being underreported, so we strongly encourage our colleagues to report any cases of individuals with a coinciding history of death by CJD and prior corneal transplantation.

Further investigation should be conducted into the transmissibility of prion disease from latent, asymptomatic donors to healthy recipients through transplantation, in order to better characterize potential routes of transmission.

\section{INTRODUCTION}

With a mortality rate of $90 \%$ within 1 year of diagnosis [1], Creutzfeldt-Jakob disease (CJD) is a rare, rapidly progressive, neurodegenerative disease caused by the abnormal accumulation of misfolded prion protein in the brain [2]. First described by German neuropathologists Hans Gerhard Creutzfeldt and Alfons Maria Jakob in 1920 [3], CJD is classified as a transmissible spongiform encephalopathy (TSE), and is characterized mainly by progressive dementia, neuron loss [4], spongiform change on histopathological sections [5], and visual disorders, with a higher prevalence in individuals over 50 years of age. Creutzfeldt-Jakob disease can be transmitted through an autosomaldominant hereditary pattern (gCJD), a sporadic pattern (sCJD), or iatrogenically (iCJD) [6]. A variant form of CJD (vCJD) thought to be caused by the agent responsible for past bovine spongiform encephalopathy outbreaks has also been identified in humans, potentially resulting from contact with contaminated meat products [7]. The latency period of the various forms of CJD has been found to have a longitude of up to 60 years, with an estimated average of 10-11 years for all forms of CJD [8]. While the 
majority of cases of CJD are acquired either sporadically $(85 \%)$ or through inheritance $(10 \%)$, there have been various reports of cases being transmitted iatrogenically through blood transfusions [9], human pituitary growth hormone (HGH) administration [10, 11], dura mater allografts $[11,12]$, surgical instrumentation [13], and transplantation of solid organs, including corneas. Though the likelihood of disease transmission through corneal transplantation has previously been calculated to be minute, risk factors and the prevalence of latent CJD in the transplant-intended donor pool have not been estimated since the analysis performed by Kennedy et al. [14] for the year 1997.

The lethality of CJD in conjunction with rising trends in corneal transplant volume, instances of multiple transplants, overall deaths due to CJD, and an aging U.S. population all warrant a contemporary risk analysis of CJD in the U.S. cornea donor pool, as performed in this article. With the development of effective screening methods of CJD in progress, the identification of donor and recipient risk factors can aid in distinguishing susceptible populations and in reporting potential cases of iatrogenic transmission of CJD through corneal transplantation globally.

\section{METHODS}

\section{Literature Review of Reported CJD Cases}

A review of the literature was conducted through PubMed, Harvard University Library Resources, Medscape, Countway Library of Medicine, JSTOR, and WorldCat databases to search for ophthalmic literature relevant to corneal transplantation and CJD-related iatrogenic transmission. The CDC Wide-ranging Online Data for Epidemiologic Research (WONDER) database was used to collect allcause mortality and CJD-caused mortality data. Statistical reports from the Eye Bank Association of America (EBAA) were used to collect data relevant to cornea donation in the United States.

Search parameters were set to include:
Creutzfeldt-Jakob Disease, Cornea CJD, CJD Risk Factors, CJD Transmission, CJD Case Reports, CJD Mortality, Corneal Donation, Prion Proteins, Cornea Anatomy and Physiology, History of Corneal Transplantation in the United States, Iatrogenic Transmission of CJD, Iatrogenic transmission of CJD through Corneal Transplantation, Ocular Tropism of Viruses, Detection of Prion Diseases, Iatrogenic Transmission of CJD through Dura Mater, Iatrogenic Transmission of CJD through Growth Hormone, Iatrogenic Transmission of Disease through Corneal Transplantation, Case reports of Iatrogenic Transmission of CJD through Corneal Transplantation in the United States, Corneal Donor Statistics in the United States, Latency Period of CJD, Detection of CreutzfeldtJakob Disease, Infectious Disease Risk Factors of Cornea Grafts, Mortality Statistics of CJD in the United States, Eye Banking and Screening for Prion Disease, Medical Standards of Cornea Donors in the United States.

The resulting articles spanned the years 1950 to 2020. Out of 1,440 articles concerning CJD, 30 articles relevant to transmission of CJD through corneal transplants are referenced in this paper. Ten cases worldwide of suspected iatrogenic transmission of CJD through corneal transplants were found among these 30 articles.

\section{Prevalence of Latent CJD-Affected Corneas in the U.S. Donor Pool in 2018}

An age-stratified analysis of the prevalence of latent CJD cases among the U.S. donor pool was carried out using CJD-specific and all-cause mortality data sourced from the U.S. Centers for Disease Control's Wide-ranging Online Data for Epidemiologic Research (CDC WONDER) database [15]. The decade-stratified CJD incidence rate in 2018 was queried in the CDC WONDER database using the ICD-10 code A81.0 for Creutzfeldt-Jakob disease. The framework for this analysis is based on prior work by Kennedy et al. [14] Corneal donor demographic data and corneal transplant data were obtained from the Eye Bank Association of America (EBAA) 2018 annual statistical report [16]. 
The prevalence of latent CJD was calculated for each 10-year age stratum between 31 and 80 years of age. This age window was chosen based on the availability of CJD-specific data from the CDC and to align with the age-stratified data reported by the EBAA. Because CJD is universally fatal, the reported death rate per million is considered equivalent to the incidence per million of symptomatic CJD. For any given 10-year age category, the prevalence per million of latent CJD was calculated as the product of CJD incidence rate in 2018, CDCreported population totals, and an estimated symptom-free disease latency of 10 years based on reported latency periods for different variants of CJD [8]. It was assumed that individuals with symptomatic CJD would be excluded from donation per eye bank criteria, while individuals with latent, undiagnosed CJD would not be prevented from donating their corneas. Although infectivity of a latent donor may be reduced compared to a symptomatic donor, we thought it was worth considering the possibility that latent donors may have the ability to transmit the prion disease through the cornea, even if the transmission rate may be low.

Age-specific all-cause mortality rates supplied by the CDC for 2018 were then applied to the estimated prevalence of CJD in each age category to determine the number of deceased individuals with latent CJD. The number of CJD-affected corneas generated is thus twice the number of deceased individuals with latent CJD. The chance that any cornea enters the relatively small pool of corneas intended for transplant is defined as the ratio of EBAA-reported corneas in the transplant pool in a given year to the total number of corneas generated by all-cause mortality in the same year. This ratio for each age category was then applied to the overall number of CJD-affected corneas generated in 2018 to determine the number of CJD-affected corneas that entered the transplant-intended pool. This analysis was performed for each age group based on EBAAreported donor demographics. It was assumed that no corneas entered the donor pool by international import given the net cornea export status of the U.S. Please refer to
Supplemental Material A for a visual depiction of this analysis.

\section{Prevalence of Latent CJD-Affected Corneas in the U.S. Donor Pool from 1979 to 2018}

Annual corneal transplant numbers from 1979 to 2018 were compiled using the EBAA statistical reports and past literature [16-18]. CJD incidence rates were queried in the CDC WONDER database using the ICD-9 code A46.1 and ICD-10 code A81.0 for the years 1979-1998 and 1999-2018, respectively. Mortality records for CJD in the U.S. were only available from 1979 onwards. An age range of 35-84 was selected based on the format of historical data. Linear regression models and trend analyses based on past historical data yielded the estimated number of corneas intended for transplant from 1979 to 2019. Please refer to Supplemental Material B for a detailed procedure outline.

From the analyses conducted, prominent risk factors for iatrogenic transmission of CJD were identified among both donors and recipients of corneal transplants.

\section{Compliance with Ethics Guidelines}

This article is based on previously conducted studies and does not contain any studies with human participants or animals performed by any of the authors.

\section{RESULTS}

\section{Summary of Global Case Reports}

Ten case reports of suspected iatrogenic transmission of CJD through cornea transplantation were found from 1974 to the present across the globe, six of which were reported in the United States [19-22], two in Germany, and two in Japan. In two reported global cases-one in the U.S. in 1974 and another in Germany in 1996both the donor and recipient were confirmed to have died of CJD (Table 1). 


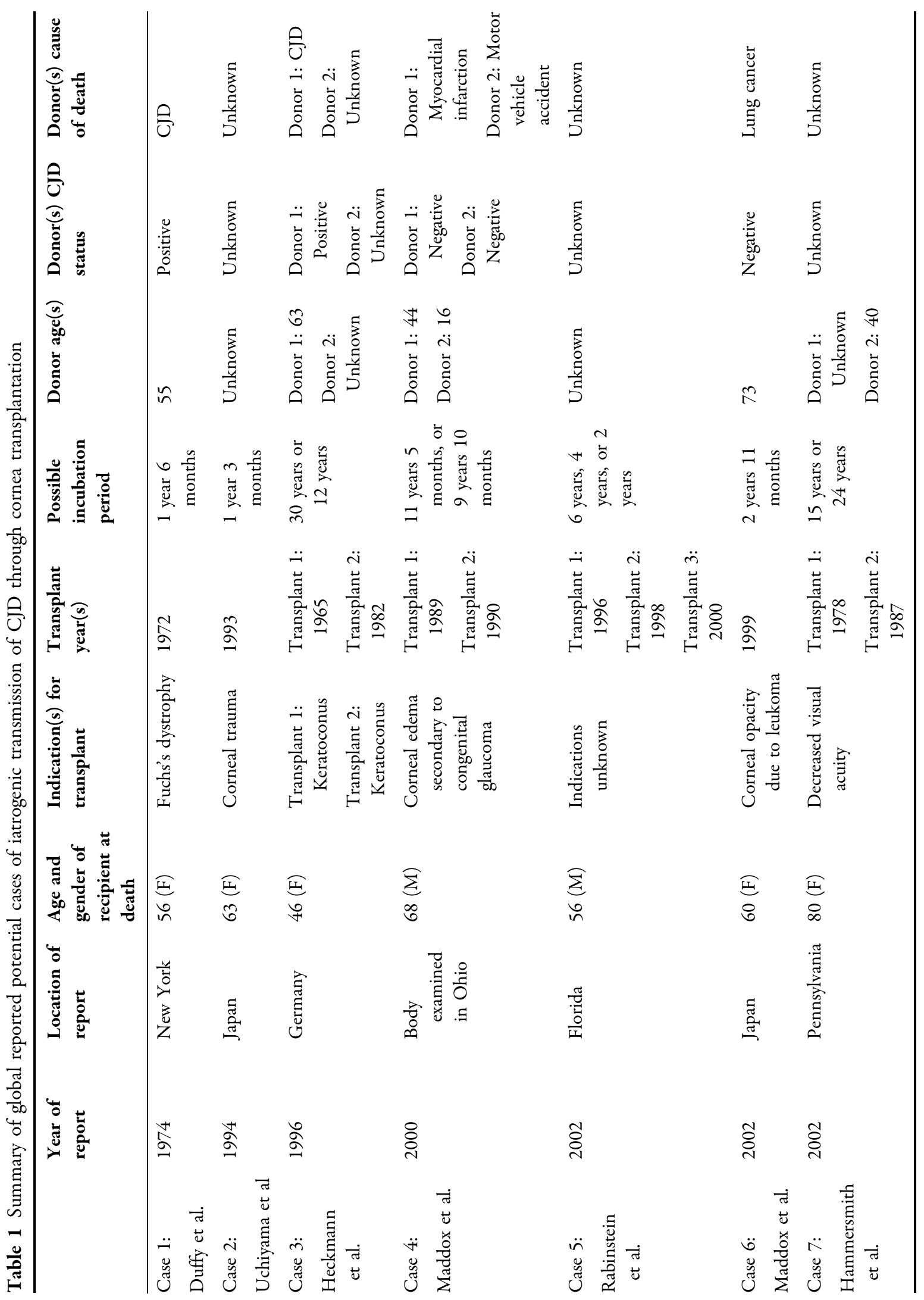




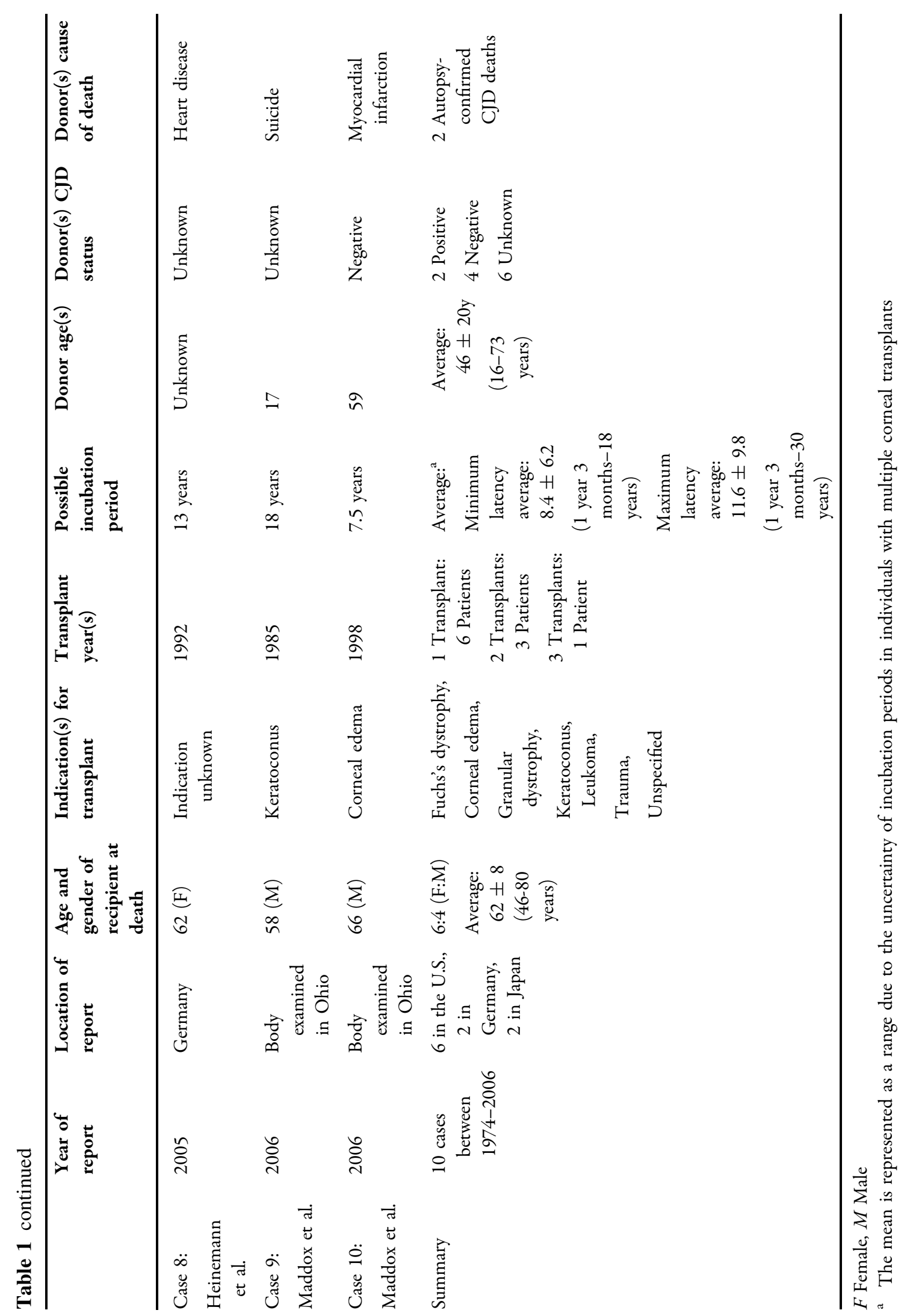


Of the 10 reported global cases, six recipients were female and four were male. The average age of the recipients at the time of death was $62 \pm 8$ years (46-80 years). The average age of identifiable donors was $46 \pm 20$ years (16-73 years). The average incubation period of global cases of iatrogenic transmission of CJD through corneal transplants was estimated to be between $8.4 \pm 6.2$ ( 1 year 3 months-18 years), as a minimum, and $11.6 \pm 9.8$ (1 year 3 months -30 years), as a maximum (Table 1 ).

Four of the 10 global cases of iatrogenic transmission ever reported were in recipients with multiple corneal transplants: three recipients had two transplants and one recipient had three past transplants (Table 1). To our best knowledge, all transplants were penetrating keratoplasties.

\section{Prevalence of CJD-Affected Corneas in 2018 Transplant-Intended Donor Pool}

Age-specific contributions to the pool of corneas recovered for intended transplant in 2018 can be found in Table 2. Individuals aged 61-70 contributed the largest proportion of CJD-affected corneas (35\%) (Table 2).

In 2018, an estimated 3.8 corneas intended for transplant came from donors between the ages of 31 and 80 with latent CJD (Table 3). These 3.8 corneas represent $0.0034 \%$ of all corneas intended for transplant in 2018 from donors aged 31-80, or one CJD-affected cornea out of every 29,500 donated corneas that entered the U.S. donor pool in 2018.

Table 2 Age-stratified cornea donor statistics, 2018

\begin{tabular}{llllll}
\hline $\begin{array}{l}\text { Age } \\
\text { group }\end{array}$ & $\begin{array}{l}\text { Deaths } \\
\text { from all- } \\
\text { cause } \\
\text { mortality }\end{array}$ & $\begin{array}{l}\text { Number of corneas } \\
\text { recovered for } \\
\text { intended transplant }^{\mathbf{a}}\end{array}$ & $\begin{array}{l}\text { Percent } \\
\text { contribution to } \\
\text { total donor pool } \\
(\%)\end{array}$ & $\begin{array}{l}\text { Percent contribution } \\
\text { to pool of donors } \\
\text { aged 31-80 (\%) }\end{array}$ & $\begin{array}{l}\text { Chance of cornea } \\
\text { entering transplant- } \\
\text { intended donor pool }^{c}\end{array}$ \\
\hline $31-40$ & 70,807 & 7621 & 6.18 & 6.8 & 0.054 \\
$41-50$ & 115,552 & 13,418 & 10.89 & 12.0 & 0.058 \\
$51-60$ & 283,804 & 29,981 & 24.33 & 26.8 & 0.053 \\
$61-70$ & 484,559 & 43,070 & 34.95 & 38.6 & 0.044 \\
$71-80$ & 624,123 & 17,613 & 14.29 & 15.8 & 0.014 \\
Total: & $1,578,845$ & 111,703 & $90.65^{\mathrm{b}}$ & 100 & 0.0354 \\
$(31-80)$ & & & & & \\
\hline
\end{tabular}

All-cause mortality statistics were obtained from the CDC WONDER database. The number of recovered corneas intended for transplant was calculated from the published EBAA statistical report data in 2018. Percent contribution from each age division was calculated using EBAA-reported donor demographic data from 2018. The total donor pool is defined as the number of recovered corneas intended for transplant in 2018. The chance that a cornea from any deceased individual in the U.S. becomes part of the pool of corneas actually recovered for intended transplant is the proportion of corneas recovered for intended transplant to the total number of corneas produced by all-cause mortality in 2018 among individuals between the ages of 31 and 80. For a visual depiction of our procedure, please refer to Electronic Supplementary Material A

a This was calculated by multiplying the total number of estimated corneas intended for transplant from donors between the ages of 31 and 80 by the percentage of cornea contribution from each age category to the overall donor pool from donors between the ages of 31 and 80

$\mathrm{b}$ The reason this is not $100 \%$ is because there are cornea donors under the age of 31 and over the age of 80 that comprise the other $9.35 \%$

c Calculated by dividing the number of corneas recovered for transplant by total number of corneas produced from all-cause mortality, which can be found by multiplying all-cause mortality by 2 ( 2 eyes/death) 
Table 3 Age-stratified prevalence of CJD in the cornea donor pool, 2018

\begin{tabular}{|c|c|c|c|c|c|c|}
\hline $\begin{array}{l}\text { Age } \\
\text { (years) }\end{array}$ & $\begin{array}{l}\text { Average CJD } \\
\text { death rate: } 2018 \\
\text { (instances per } \\
1,000,000)\end{array}$ & $\begin{array}{l}\text { All-cause } \\
\text { death rate } \\
\text { (instances per } \\
100,000 \text { ): } \\
2018\end{array}$ & $\begin{array}{l}\text { Prevalence } \\
\text { of latent } \\
\text { CJD } \\
\text { (cases): } \\
2018\end{array}$ & $\begin{array}{l}\text { Deceased } \\
\text { individuals } \\
\text { with latent } \\
\text { CJD (cases): } \\
2018^{a}\end{array}$ & $\begin{array}{l}\text { Total corneas } \\
\text { from donors } \\
\text { with latent } \\
\text { CJD: } 2018^{a}\end{array}$ & $\begin{array}{l}\text { Corneas from donors } \\
\text { with latent CJD } \\
\text { recovered for intended } \\
\text { transplant: } 2018^{\mathrm{a}}\end{array}$ \\
\hline $31-40$ & 0 & 162.03 & 0 & 0 & 0 & 0 \\
\hline $41-50$ & 0.47 & 285.58 & 190 & 0.5 & 1.1 & $0.06(0.1)$ \\
\hline $51-60$ & 1.59 & 662.70 & 680 & 4.5 & 9.0 & $0.477(0.5)$ \\
\hline $61-70$ & 4.86 & 1294.97 & 1820 & 23.6 & 47.1 & $2.07(2.1)$ \\
\hline $71-80$ & 6.13 & 2752.78 & 1390 & 38.3 & 76.5 & $1.07(1.1)$ \\
\hline $\begin{array}{l}\text { Total: } \\
\text { Age } \\
31-80\end{array}$ & 2.19 & 843.95 & 4080 & 66.9 & 133.8 & 3.8 \\
\hline
\end{tabular}

The CJD death rate (incidence rate) is presented for the specified age groups based on available data from the CDC WONDER mortality database for 2018. All-cause mortality rate data are similarly sourced from the CDC WONDER mortality database for 2018. The prevalence of Latent CJD is calculated from the CJD incidence rate using an asymptomatic disease latency of 10 years. The number of deceased individuals with latent CJD in 2018 is thus the estimated 2018 prevalence of CJD multiplied by the published general all-cause mortality rate. Each deceased individual with CJD is assumed to have two corneas theoretically available for donation. The number of these corneas that are actually recovered for intended transplant is the number of latent CJD-affected corneas multiplied by the chance that a cornea from any deceased individual enters transplant-intended donor pool (Table 2). For a visual depiction of our procedure, please refer to Electronic Supplementary Material A

${ }^{\text {a }}$ Numbers were rounded. These rounded numbers were added to calculate totals

\section{Historical Analysis from 1979 to 2018}

In the data compilation of corneal transplantation and CJD-related mortality in the United States from 1979 to 2018, we found that corneal transplants performed in the U.S. have generally been on a steady rise, with a plateau between 1990 and 2005, when the U.S. began exporting corneas internationally (Fig. 1). The number of mortalities due to CJD have doubled within the last 20 years (Fig. 2). Between 1979 and 2018, an estimated 47 corneas entered the transplant-intended donor pool from individuals between the ages of 35 and 84 with latent CJD (Table 4, Fig. 3). This translates to roughly one affected cornea for every 30,000 corneas used in transplants between 1979 and 2018.

\section{DISCUSSION}

Between 1961 and 2018, 1,954,600 corneal transplants have been performed with corneas from U.S. eye banks [16]. Corneal transplantation procedures are becoming more routine than ever, with a $60 \%$ increase in endothelial keratoplasty (EK) procedures, including Descemet stripping endothelial keratoplasty (DSEK) and Descemet membrane endothelial keratoplasty (DMEK), within just the last 10 years. As the largest exporter of corneal tissue, the United States exported over one third of donated cornea tissue to countries across the globe in 2018 [16], exemplifying the globalization of corneal transplantation. With the number of corneal transplants rising each year in the United States, and with dozens of countries using U.S. corneal tissue for transplantation, an understanding of 


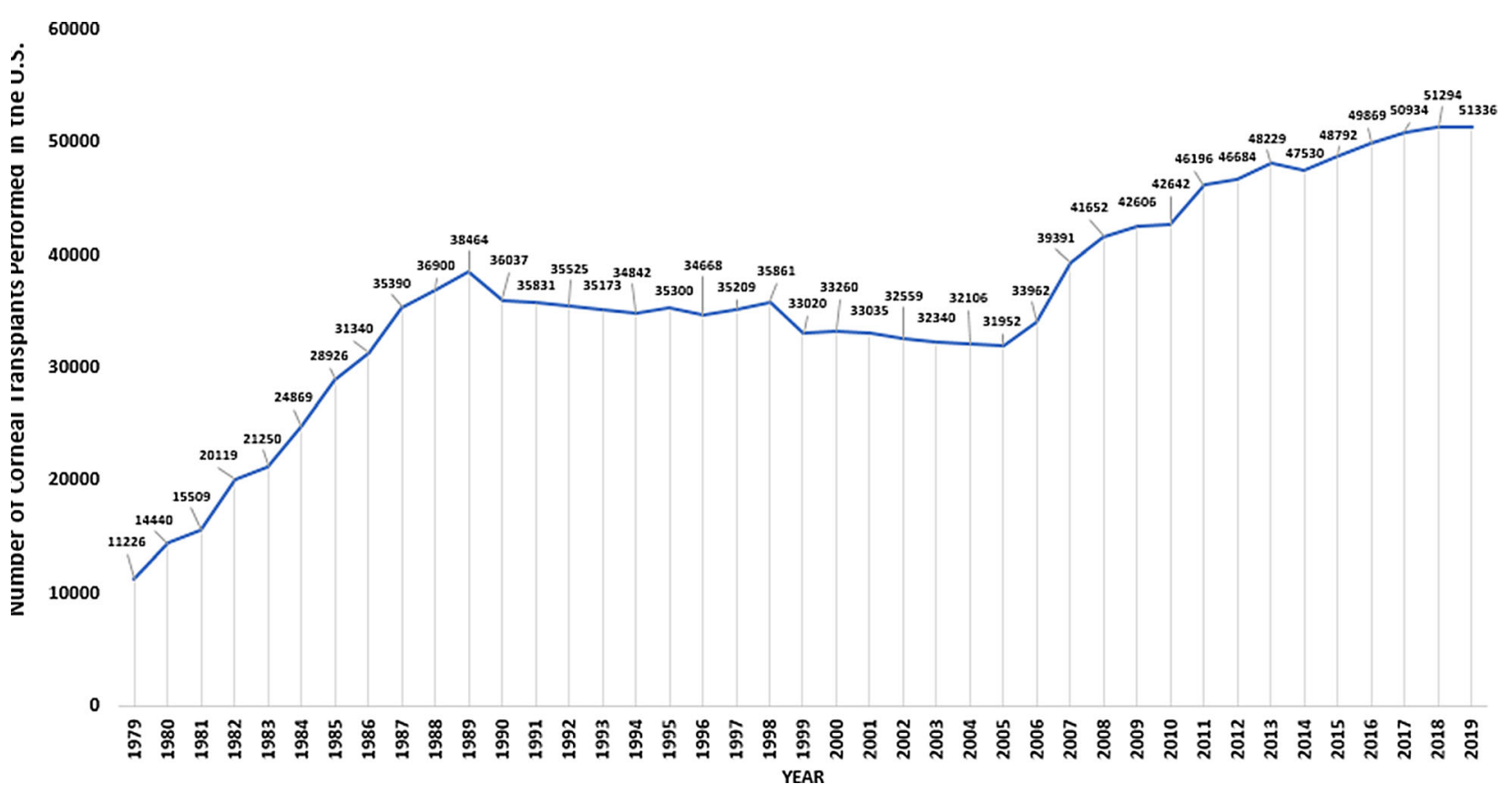

Fig. 1 Number of cornea transplants performed annually in the United States from 1979 to 2019. These totals do not include transplants conducted outside the U.S. with

the potential risk of iatrogenic transmission from donor to host through corneal tissue is essential, given its life-threatening implications. Creutzfeldt-Jakob disease is a debilitating, fatal prion disease that has been shown to cause infection in organ tissues, with reported cases of suspected iatrogenic transmission through neurosurgery [23], kidney transplants [24], and corneal transplantation in human beings. Although case reports of CJD transmission via corneal transplantation have been sporadic and infrequent, the severity of this disease, the greatly varying latency period, the possibility of unreported cases, and increased screening measures for CJD transmission risk all warrant a proper analysis of estimating the number of corneas in the annual U.S. donor pool from individuals with undiagnosed, latent CJD.

Both our 2018 analysis and our historical analysis from 1979 to 2018 yielded the same result: an estimated 1 in every 30,000 transplants in the U.S. will be performed with a cornea from a donor between the ages of 31 and 80 with latent CJD. Although we estimated 3.8 corneas from donors with latent CJD entering exported U.S. tissue. Data were sourced from the Eye Bank Association of America (EBAA) statistical reports

the U.S. donor pool in 2018, a prior analysis performed by Kennedy et al. in 1997, when the number of cornea donations was approximately half the present number (Table 4), yielded an estimate of 2.4 corneas from donors with latent CJD entering the U.S. donor pool annually.

Increases in cornea donation and size of the U.S. population have not significantly altered the risk of tissue recipients being exposed to CJD. Over the last decade, the CJD incidence rate has remained relatively stagnant; as such, employing routine molecular screening modalities to capture undetected CJD in banked donor corneas is still not recommended, despite the improved efficacy and cost of these assays. As a result, the most pragmatic course of action is to identify risk factors for iatrogenic transmission of CJD through corneal transplants from historical data. These risk factors could aid in more focused and rapid investigations into potential cases.

Based on the results of our analysis, age was identified as a primary donor risk factor for a deceased individual with latent CJD contributing a cornea to the transplant-intended U.S. 


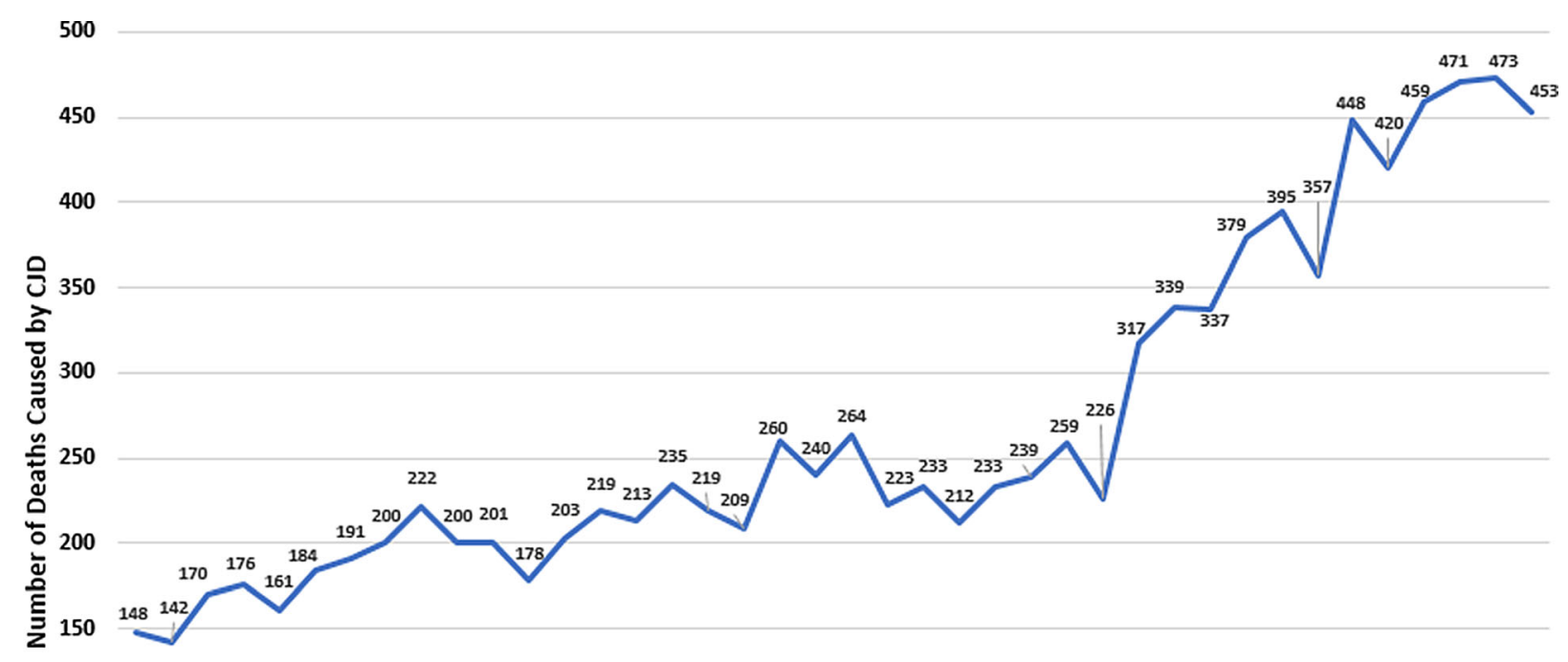

100

50

0

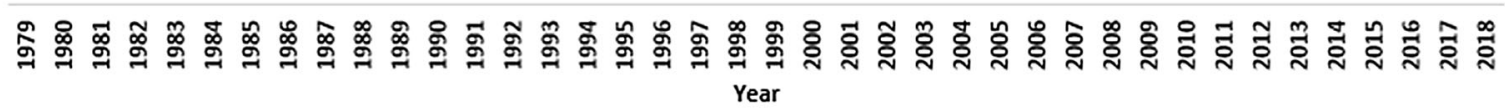

Fig. 2 Annual number of deaths caused by CJD in the United States from 1979 to 2018. These totals include deaths among individuals of all ages. Mortality data were sourced from the CDC WONDER database

donor pool. From 2007 to 2017, there was a $34 \%$ increase in the population of individuals over 65 years of age in the U.S. [25]. As a result, more elderly individuals are donating corneas, receiving cornea transplants, and dying of CJD, as reflected by the doubling of the number of CJD mortalities within the past 20 years. Of the 3.8 corneas from donors with latent CJD estimated to enter the transplant-intended U.S. donor pool in 2018, 84\% (3.2) were contributed by individuals aged $61-80$ (Table 3 ). Our findings are congruent with those of Kennedy et al., who found that the highest-risk donor age group for iatrogenic transmission of CJD was 60-69 years. This can be accounted for by the fact that an estimated $92 \%$ of deaths among individuals between $31-80$ with latent CJD occur in individuals over 60 years old. For other non-PKP uses of corneal tissue, such as DMEK, the average age of donors is 70 [26]. Recipients of corneas from donors over 60 years of age are at greater risk of transmission of CJD than those receiving corneas from donors under the age of 60.

Thus, of the donors reported in the 10 global case reports, two donors were under the age of 20 (Table 1), which significantly diminishes the likelihood that they died of a prion disease. Based on this, it is highly unlikely that these younger donors transmitted CJD to the recipients through corneal transplant; these cases could likely be due to coincidental sporadic CJD in corneal transplant recipients. For the two global cases in which both the donor and the recipient died of CJD, the donors were 55 and 63 years of age, the latter of which was at greater risk of transmitting CJD through the cornea than younger donors due to old age (Table 1).

A contributing risk factor in recipients is having received multiple transplants in a lifetime, especially if their donors were elderly. In $2018,12.3 \%$ of all corneal transplants performed, or 5966 procedures, were repeat transplants [16]. It is worth noting that individuals 


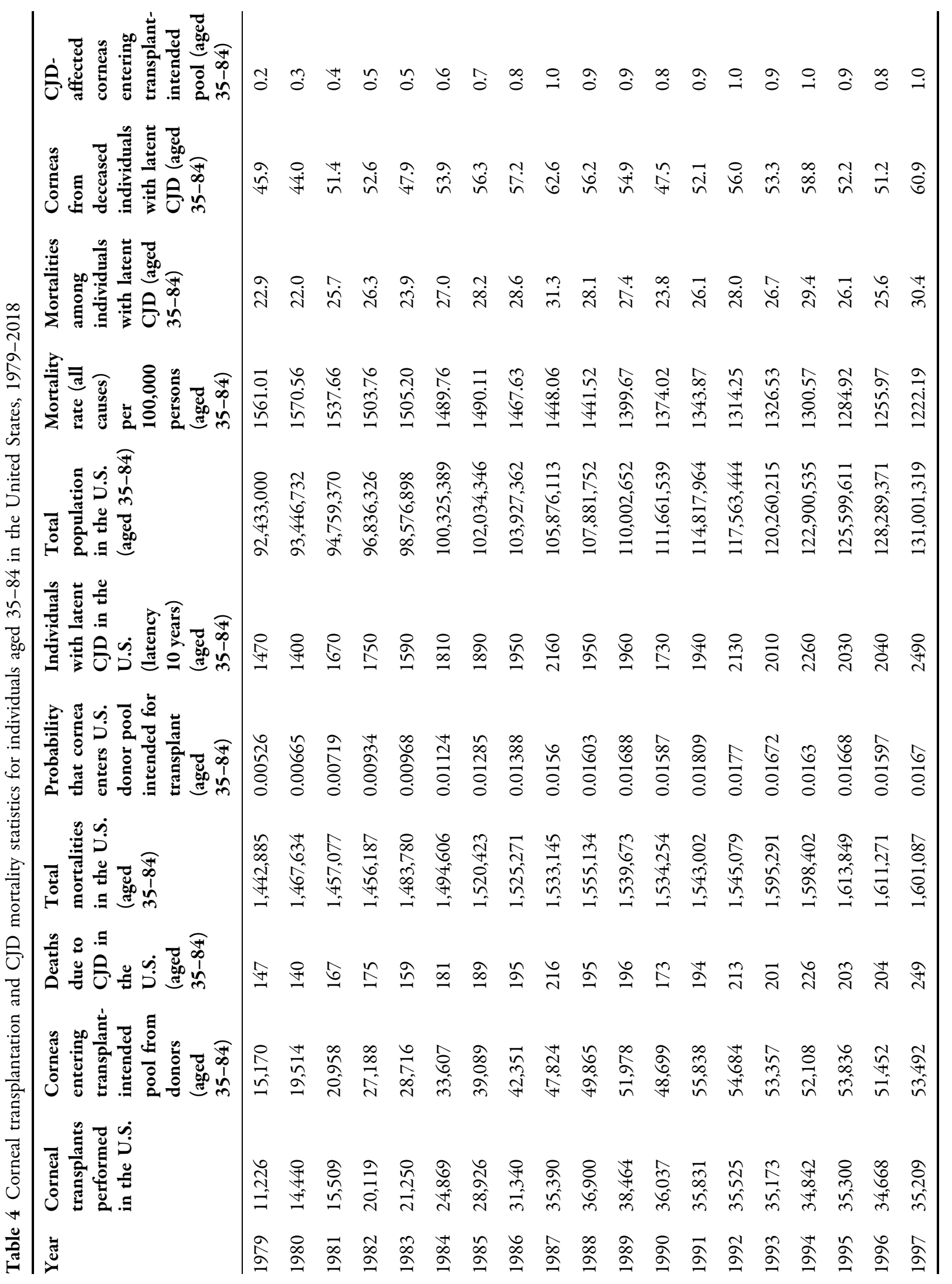




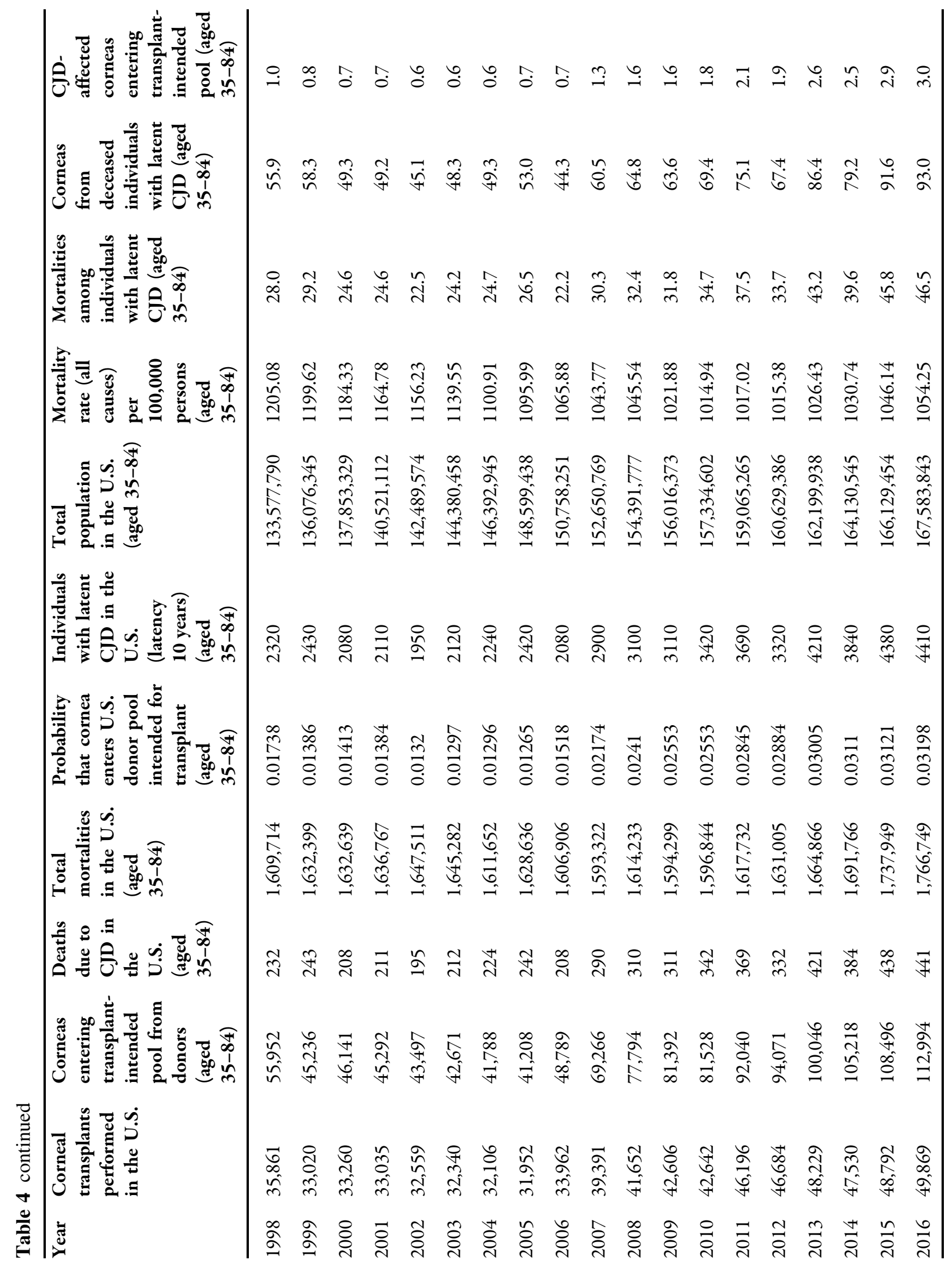




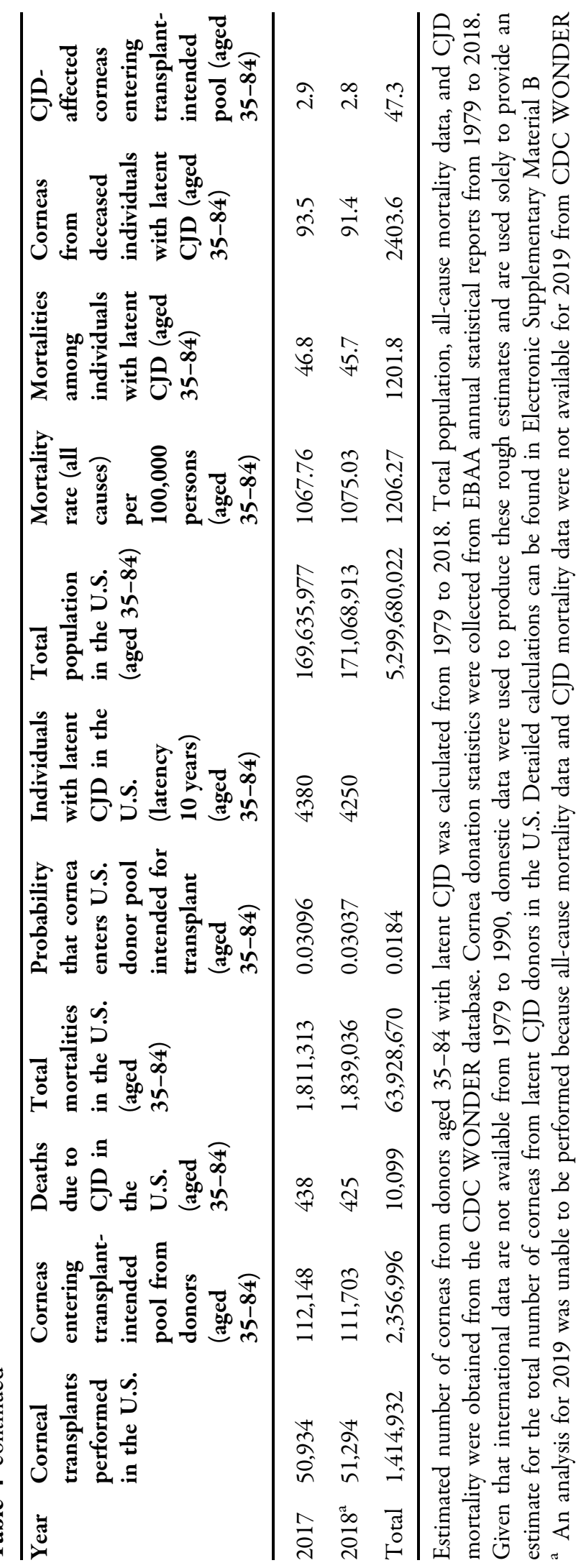




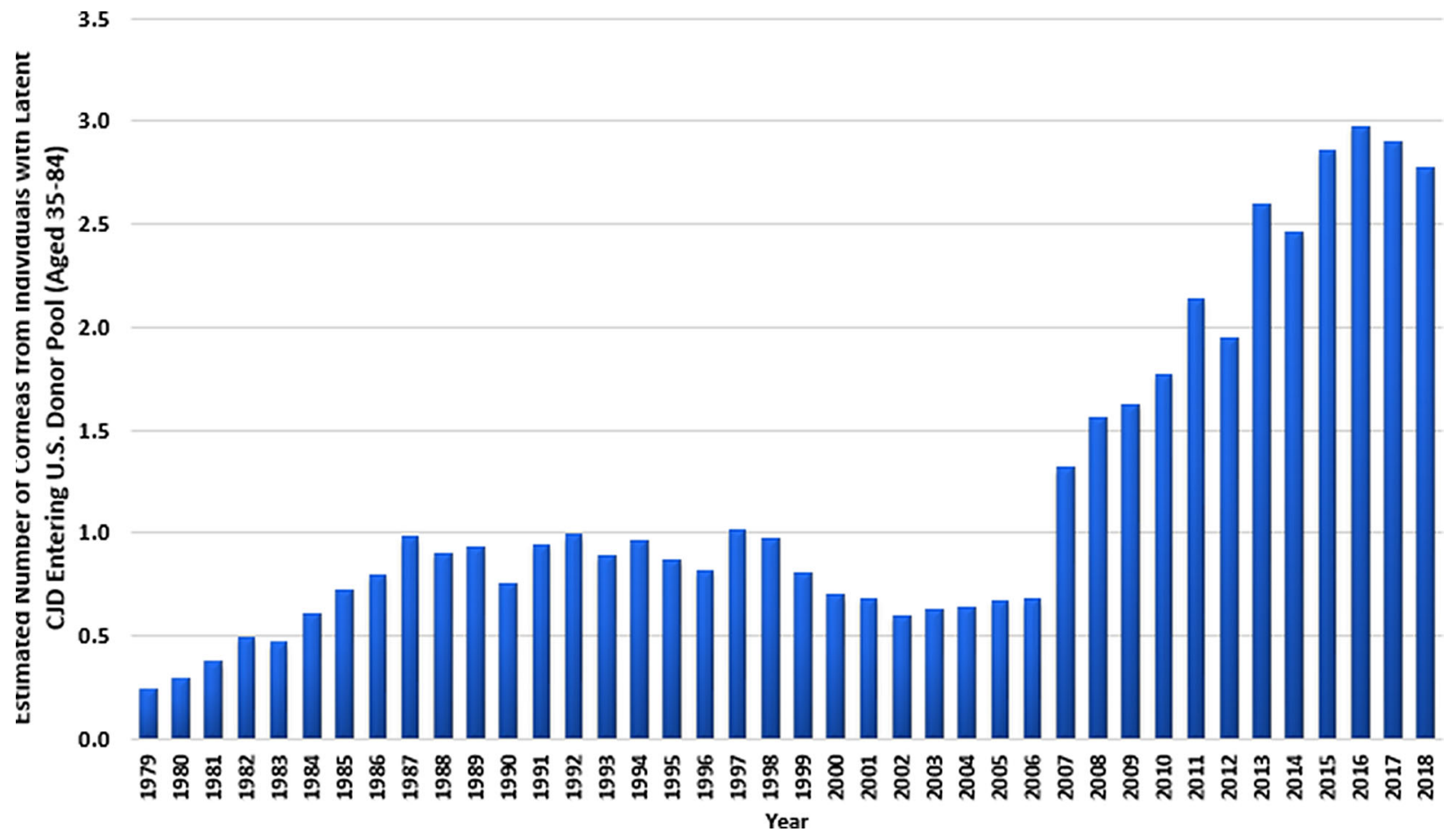

Fig. 3 Estimated annual number of corneas from donors with latent CJD aged 35-84 entering the U.S. transplantintended pool from 1979 to 2018. These estimates were calculated based on the data in Table 4, for which the CDC WONDER database and the EBAA statistical reports were used. Values are rounded to the nearest tenth.

who have had multiple corneal transplants will have an increased risk of undergoing a transplant with a cornea from an individual with latent CJD. For example, if a patient undergoes a single corneal transplant in their lifetime, based on 2018 data, the overall probability that the cornea used in their transplant was from a donor with latent CJD between the ages of 31 and 80 is $0.0034 \%(3.8 / 111,703)$, or one cornea from a donor with latent CJD of every 29,500 corneas intended for transplant. However, if this patient undergoes a repeat corneal transplant, the overall likelihood that one of their donors was affected with latent CJD is doubled and becomes $0.0068 \%$, or a 1 in 14,700 likelihood. A third corneal transplant would increase this likelihood to $0.00102 \%$, or a 1 in 9800 likelihood. The risk becomes $0.005 \%$ for one transplant if the donor is over 60 years of age; for multiple transplants, this probability
Although our age-stratified calculations (Tables 2, 3) determined an estimated number of 3.8 in 2018, this data set is based on a slightly different age range- -35 to 84 , compared with 31 to 80 - which could explain this discrepancy

increases accordingly. These risk factors can be utilized to more easily identify candidates in which the likelihood of iatrogenic transmission may be heightened. It is also worth noting that penetrating keratoplasties were the main form of corneal transplant performed among these 10 cases; procedures such as DMEK and DSEK, which are becoming increasingly popular, do not the involve transplant of the entire cornea, and may result in reduced transmissibility rates, as less infected tissue is being used. Thus, penetrating keratoplasties may potentially pose a greater risk of transmission than other transplant procedures; however, there is insubstantial evidence to confirm this hypothesis as a definite risk factor.

Although the prevalence of CJD in the corneal donor pool is exceedingly low, the global reporting of a mere 10 cases of potential CJD transmission through corneal donation since 
1974, with no cases documented after 2006, is lower than expected. Maddox et al. previously estimated that a case of CJD in corneal transplant recipients would be expected to occur once every 1.5 years [19]. At this rate, we would estimate there to have been eight potential cases since the last reported case in 2006. Thus, underreporting of cases of overlapping histories of death by CJD and prior corneal transplantation is likely occurring.

Of the 47 estimated CJD-affected corneas entering the U.S. donor pool since 1979 (Table 4), five of them, or $10.6 \%$, were reported to have potentially caused CJD infection in recipients after transplantation (Table 1 ). Based on this small number of reported cases, and the lack of sufficient donor data for most of these reported cases, it is likely that transmission through the cornea does not occur in $100 \%$ of cases of corneal transplantation from a donor with latent CJD, which is supported in an article written by Tullo et al. [27] It is more likely that if these recipients did develop the infection from CJD-affected corneas, they may still be in the latency phase of the infection, which can continue for decades. Thus, they may begin to display symptoms in years to come, and because of this, there is no way of truly estimating an accurate transmissibility risk of CJD through the cornea other than through histopathological examination. Because the rate of transformation between receiving a CJD-affected cornea and developing latent CJD is unknown, it is difficult to distinguish whether the low number of reported cases is driven primarily by a low disease conversion rate, underreporting, or a combination of both.

Additionally, there is an apparent lack of geographical diversity among reported cases. Of the four internationally reported cases, two were in Japan and two were in Germany (Table 1), both countries with heightened awareness of CJD due to past history. Because of the wide transmission of CJD through German Lyodura brand dura mater cadaveric grafts in the 1970s and 1980s, attention was called to iatrogenic transmission of CJD in Germany. Similarly, Japan has reported an unusually high prevalence of dura mater-related CJD transmission, primarily due to the use of these German
Lyodura grafts before 1987 [11, 12]. Cases of iatrogenic transmission of CJD through corneal transplantation may have been present in other countries. However, due to a lack of awareness of iatrogenic transmission, these cases most likely have not been reported, perhaps because cornea transplantation history of individuals who died of CJD was likely not investigated.

However, the reality that CJD was confirmed in both the donor and host in only 2 out of 10 globally reported cases (Table 1) lends credence to the former hypothesis of low transmissibility. To simply report overlapping histories of death by CJD and prior transplantation, although important for capturing possible cases, in no way evidences a causal link between the two. In fact, the molecular mechanisms underlying how CJD might spread from an implanted cornea to the central nervous system of the host remain entirely unclear. Experiments in guinea pigs wherein CJD-affected corneal tissue was implanted directly into the anterior chamber have shown the potential for misfolded prion proteins to diffuse through the vitreous to cause an ascending CNS infection [28]. However, these experimental conditions do not fully model the incorporation of live tissue as in a human corneal transplant.

Past studies of sporadic CJD in humans determined that prion seeds were present in ocular tissues such as the choroid, sclera, optic nerve, vitreous, lens, muscle, and the cornea [29]. Retrograde transmission from brain to cornea has been hypothesized to occur by the transport of misfolded prion protein by cranial nerves; the prions spread from the brain into the retina by axonal transport in the optic nerve, accumulating in small nerves in the cornea [29]. The transmission of iatrogenic CJD from the cornea to the brain would follow an anterograde pattern, potentially through axonal transport of exogenous misfolded prion protein to the midbrain via retinal ganglion cells $[30,31]$. The overall mechanism may be that misfolded prion proteins from the CJD-affected transplanted cornea could spread to the retina of the recipient through vitreous humors; there, it accumulates in retinal ganglion cells, and axonal transport by these retinal ganglion cells 
to the midbrain would result in CJD transmission.

Prion diseases have usually been diagnosed through post-mortem histopathological examination of brain tissue, which has been neither cost- nor time-effective [32]. Because genetically screening the entire donor population may be infeasible, it is important to focus on ongoing development of cost-effective, efficient screening measures for prion disease. Besides genetic screening for familial CJD, screening for prion disease, including all forms of CJD, should be routine in donor examination, or general wellness exams, especially for potential donors over the age of 60 . Recently, there have been efforts to improve the efficacy of post-mortem CJD detection. In 2017, Gregori et al. [33] proposed a method of rapid testing for CJD in cornea donors, using commercial testing combined with rapid sample collection, which proved to be able to detect even low concentrations of prion protease-resistant abnormal prion protein, or $\mathrm{PrP}^{\mathrm{TSE}}$. $\mathrm{PrP}^{\mathrm{TSE}}$ testing could potentially be used to detect CJD even during its early stages in the cornea, enhancing the safety of corneal transplants. Strides have been made in the innovation of pre-mortem detection of prion disease as well. As of 2018, diagnostic tests utilizing protein misfolding cyclic amplification (PMCA) for pre-mortem detection of prion disease, specifically vCJD, through the blood were being developed. Pre-mortem tests, if applicable to multiple variants of CJD, including sporadic CJD, could assist in more accurate diagnosis of individuals with pre-symptomatic, latent CJD, potentially eliminating the possibility of iatrogenic transmission through organ tissues [34].

It may be worthwhile to further investigate the transmission rate of CJD through corneal transplantation from a latent, CJD-affected donor into a healthy recipient and confirm the presence of CJD along the ocular route to the brain through a histopathological examination, rather than by waiting for symptoms to present. Although previous studies have been conducted with less sensitive immunohistochemical assays, with the highly sensitive, rapid techniques of prion protein detection present nowadays, this study could be conducted with higher accuracy and serve to better illustrate the mechanisms by which CJD may be transmitted from the cornea to the brain.

Our methodology had several limitations. We utilized CDC WONDER as our sole mortality database for both CJD and all-cause mortality. There exist multiple databases that report varying numbers of CJD deaths and we acknowledge that CDC WONDER may underreport the number of annual CJD cases; however, we selected CDC WONDER due to the use of this database in past analyses. This analysis does not include individuals with symptomatic undiagnosed CJD. Because the average time to diagnosis after symptom onset is approximately 6 months [35], this would contribute a minimal number of donors.

For the global case report summary, there were cases in which the recipient had received multiple past corneal transplants, leading us to estimate ranges for the average incubation period, rather than an exact average. Many donors were unable to be identified; thus, the cause of death of donors was not confirmed.

For all calculations, we used an incubation period of 10 years. The CJD incubation period has been found to last as long as 60 years; because there has been no statistical analysis performed to determine the incubation period for all forms of CJD confidently, we made an estimate based on literature review and values used in other analyses. We also could not conduct any analyses for donors younger than 31 because there was insufficient mortality data from CDC WONDER. We were given the total number of corneas intended for transplant, but we were not given age-stratified data for this number, so we estimated this based on overall percent contributions of each age group to the total U.S. donor pool in 2018.

For the historical data analysis, we were unable to calculate values for the donor age group 31-80, because prior to 1999, CDC WONDER reported mortality data only in 10-year age stratifications starting at age 25 . Thus, we used the age group 35-84. We were also unable to find the number of corneas intended for transplant in the U.S. prior to 2011, which is why we used a linear regression and trend analysis to estimate that number. Because EBAA only reports data in 10-year age 
intervals (example: $31-40$ ), we assumed that the total number of corneas intended for transplant from donors aged $31-80$ would be similar to the number from donors aged 35-84.

\section{CONCLUSION}

Although contemporary and past statistical analyses have shown that the prevalence of CJD-affected corneas entering the U.S. donor pool is low, we conclude that potential cases of CJD occurrence in both the donor and recipient of corneal transplants are underreported globally. For the sake of advancing neurological research in this field, all mortalities from CJD in the United States, and even globally, should be investigated for past corneal transplant history. If it is discovered that an individual who dies from CJD also has a history of corneal transplants, the cause of death of their cornea donor(s) should be investigated and reported. Similarly, if recipients of corneal transplants are diagnosed with CJD sometime within their lifetime, this should be reported immediately, and any potential recipient of the other cornea from the same donor pair should be promptly alerted and tested for any sign of prion disease. The advanced age of donors and multiple transplant history of recipients both present increased risk for iatrogenic transmission of CJD through corneal tissue. Advances in screening methods, especially among the elderly, could result in more cost-effective, rapid detection of prion disease in latent donors prior to transplantation. With corneal transplants and the number of deaths caused by CJD on the rise, further research should be conducted to investigate the pathology and detection methods behind the transmission of Creutzfeldt-Jakob disease through corneal tissue, as it can lead to a potentially life-threatening outcome of transplantation.

\section{ACKNOWLEDGEMENTS}

Funding. This study was funded by an unrestricted grant from Research to Prevent
Blindness (RPB), 360 Lexington Avenue, 22nd Floor New York, NY 10017. No support was received for the publication of this article.

Authorship. All named authors meet the International Committee of Medical Journal Editors (ICMJE) criteria for authorship for this article, take responsibility for the integrity of the work as a whole, and have given their approval for this version to be published.

Disclosures. Tanisha Martheswaran, Jordan D. Desautels, Majid Moshirfar, Kathryn M. Shmunes, Yasmyne C. Ronquillo and Phillip C. Hoopes have no conflicts of interest related to this work.

Compliance with Ethics Guidelines. This article is based on previously conducted studies and does not contain any studies with human participants or animals performed by any of the authors.

Data Availability. All data generated or analyzed during this study are included in this published article/as supplementary information files.

Open Access. This article is licensed under a Creative Commons Attribution-NonCommercial 4.0 International License, which permits any non-commercial use, sharing, adaptation, distribution and reproduction in any medium or format, as long as you give appropriate credit to the original author(s) and the source, provide a link to the Creative Commons licence, and indicate if changes were made. The images or other third party material in this article are included in the article's Creative Commons licence, unless indicated otherwise in a credit line to the material. If material is not included in the article's Creative Commons licence and your intended use is not permitted by statutory regulation or exceeds the permitted use, you will need to obtain permission directly from the copyright holder. To view a copy of this licence, visit http://creativecommons.org/licenses/by$\mathrm{nc} / 4.0 /$. 


\section{REFERENCES}

1. Mcelvanney AM, Boodhoo MG. Creutzfeldt-Jakob disease presenting with visual disturbance. Eye. 1999;13(5):693-5.

2. Head MW, Northcott V, Rennison K, Ritchie D, Mccardle L, Bunn TJR, et al. Prion protein accumulation in eyes of patients with sporadic and variant Creutzfeldt-Jakob disease. Invest Ophthalmol Vis Sci. 2003;44(1):342.

3. Lanska DJ. Etymologia: Creutzfeldt-Jakob Disease. Emerg Infect Dis 2017; 23(10):1760-1761.

4. Iwasaki Y. Creutzfeldt-Jakob disease. Neuropathology. 2016;37(2):174-88.

5. Hamaguchi T, Noguchi-Shinohara M, Nozaki I, Nakamura Y, Sato T, Kitamoto T, et al. The risk of iatrogenic Creutzfeldt-Jakob disease through medical and surgical procedures. Neuropathology. 2009;29(5):625-31.

6. Armitage $\mathrm{WJ}$, Tullo $\mathrm{AB}$, Ironside JW. Risk of Creutzfeldt-Jakob disease transmission by ocular surgery and tissue transplantation. Eye. 2009;23(10):1926-30.

7. Mackay GA, Knight RS, Ironside JW. The molecular epidemiology of variant CJD. Int J Mol Epidemiol Genet. 2011;2(3):217-27.

8. Huillard d'Aignaux J, Costagliola D, Maccario J, et al. Incubation period of Creutzfeldt-Jakob disease in human growth hormone recipients in France. Neurology. 1999;53(6):1197-201.

9. Brown P, Brandel JP, Sato $\mathrm{T}$, et al. Iatrogenic Creutzfeldt-Jakob disease, final assessment. Emerg Infect Dis. 2012;18(6):901-7.

10. Lewis AM, Yu M, Dearmond SJ, Dillon WP, et al. Human growth hormone-related iatrogenic Creutzfeldt-Jakob disease with abnormal imaging. Arch Neurol. 2006;63(2):288.

11. Hamaguchi T, Sakai K, Noguchi-Shinohara M, et al. Insight into the frequent occurrence of dura mater graft-associated Creutzfeldt-Jakob disease in Japan. J Neurol Neurosurg Psychiatry. 2013;84(10):1171-5.

12. Ae R, Hamaguchi T, Nakamura Y, et al. Update: Dura Mater Graft-Associated Creutzfeldt-Jakob Disease-Japan, 1975-2017. MMWR Morb Mortal Wkly Rep. 2018;67(9):274-8.

13. Bastian FO. The case for involvement of spiroplasma in the pathogenesis of transmissible spongiform encephalopathies. J Neuropathol Exp Neurol. 2014;73(2):104-14.
14. Kennedy RH. Eye banking and screening for Creutzfeldt-Jakob disease. Arch Ophthalmol. 2001;119(5):721.

15. Centers for Disease Control and Prevention. CDC WONDER. https://wonder.cdc.gov/.

16. 2018 Eye Banking Statistical Report: U.S. Eye Banking Statistics Reported by U.S. Banks. Eye Bank Association of America, Washington, DC, 2019.

17. 2019 Eye Banking Statistical Report: U.S. Eye Banking Statistics Reported by U.S. Banks. Eye Bank Association of America, Washington, DC, 2020.

18. Chu $\mathrm{W}$. The past twenty-five years in eye banking. Cornea. 2000;19(5):754-65.

19. Maddox RA, Belay ED, Curns AT, Zou W-Q, Nowicki $S$, Lembach RG, et al. Creutzfeldt-Jakob disease in recipients of corneal transplants. Cornea. 2008;27(7):851-4.

20. Rabinstein AA, Whiteman ML, Shebert RT. Abnormal diffusion-weighted magnetic resonance imaging in Creutzfeldt-Jakob disease following corneal transplantations. Arch Neurol. 2002;59(4):637-9.

21. Hammersmith KM, Cohen EJ, Rapuano CJ, Laibson PR. Creutzfeldt-Jakob disease following corneal transplantation. Cornea. 2004;23(4):406-8.

22. Duffy P, Wolf J, Collins G, DeVoe AG, Streeten B, Cowen D. Letter: Possible person-to-person transmission of Creutzfeldt-Jakob disease. N Engl J Med. 1974;290(12):692-3.

23. Mayor S. UK government advises tighter measures to reduce risk of CJD transmission during neurosurgery. BMJ. 2003;326(7388):517.

24. Nwadike E, Kannabhiran D, Calderon K, et al. Creutzfeldt-Jakob disease (CJD) in an adult kidney transplant patient. Am J Kidney Dis. 2014;63(5): B83.

25. Department of Health and Human Services (2018). 2018 Profile of Older Americans; 2018 ASI 4004-43

26. Schaub F, Enders P, Zachewicz J, et al. Impact of donor age on descemet membrane endothelial keratoplasty outcome: evaluation of donors aged 17-55 years. Am J Ophthalmol. 2016;170:119-27.

27. Tullo A, Buckley R, Kelly T, Head M, Bennett P, Armitage W, Ironside J. Transplantation of ocular tissue from a donor with sporadic Creutzfeldt-Jakob disease. Clin Exp Ophthalmol. 2006;34(7):645-9.

28. Manuelidis EE. Transmission of Creutzfeldt-Jakob disease from man to the guinea pig. Science. 1975;190(4214):571-2. 
29. Orrù CD, Soldau K, Cordano C, et al. Prion seeds distribute throughout the eyes of sporadic Creutzfeldt-Jakob disease patients. mBio. 2018;9(6): e02095-e2118.

30. Butowt R, Abdelraheim S, Brown DR, Bartheld CSV. Anterograde axonal transport of the exogenous cellular isoform of prion protein in the chick visual system. Mol Cell Neurosci. 2006;31(1):97-108.

31. Butowt R, Davies P, Brown DR. Anterograde axonal transport of chicken cellular prion protein $(\mathrm{PrPc})$ in vivo requires its N-terminal part. J Neurosci Res. 2007;85(12):2567-79.

32. Kübler E, Oesch B, Raeber AJ. Diagnosis of prion diseases. Br Med Bull. 2003;66:267-79.
33. Gregori L, Serer AR, McDowell KL, Cervenak J, Asher DM. Rapid testing for Creutzfeldt-Jakob disease in donors of cornea. Transplantation. 2017;101(4):e120-e124124.

34. Bougard D, Bélondrade M, Mayran C, et al. Diagnosis of methionine/valine variant CreutzfeldtJakob disease by protein misfolding cyclic amplification. Emerg Infect Dis. 2018;24(7):1364-6.

35. Hogan RN, Brown P, Heck E, Cavanagh HD. Risk of prion disease transmission from ocular donor tissue transplantation. Cornea. 1999;18:2-11. 